\title{
Chronic Myelomonocytic Leukemia in Remission
}

National Cancer Institute

\section{Source}

National Cancer Institute. Chronic Myelomonocytic Leukemia in Remission. NCI

Thesaurus. Code C8616.

Chronic myelomonocytic leukemia not growing; responding to treatment. 